\title{
THE FEATURES THAT SHAPE FEAR: HOW EMOTIONAL INTENSITY AND THREAT RELEVANCE INTERACT TO GUIDE FEAR GENERALIZATION
}

Mason McClay ${ }^{1}$, Augustin C. Hennings ${ }^{2}$, Alex Reidel ${ }^{1}$, \& Joseph E. Dunsmoor ${ }^{1,2}$

1. Department of Psychiatry, Dell Medical School, University of Texas at Austin

2. Institute for Neuroscience, University of Texas at Austin

Correspondence: Joseph E Dunsmoor, joseph.dunsmoor@austin.utexas.edu 


\begin{abstract}
The amount of fear of a potential threat is oftentimes proportional to the overlap in shared features with a known threat. An adaptive threat learning system should therefore extract the most relevant feature of a known threat to help successfully detect and appropriately respond to potential threats in the future. But what if the most salient feature of a known threat is emotionally positive? Here, we asked whether fear generalization can be guided be positive emotional features. We first paired a slightly happy (Experiment 1) or fearful (Experiment 2) face with an electrical shock. We then tested fear generalization to modified face stimuli of the same identity exhibiting more or less happiness or fear expression. Both experiments yielded biased physiological arousal to a face stimulus with the most exaggerated emotional expression. These findings suggest that overlap of positive emotional features extracted from a known threat can guide biased fear generalization.
\end{abstract}

Keywords: emotion learning, fear, fear generalization, generalization learning, Pavlovian conditioning 


\section{INTRODUCTION}

When confronted with potential threats, animals can adapt behaviors that aid in survival by relying on prior experiences with similar threats (Dunsmoor, Prince, Murty, Kragel, \& LaBar, 2011; Onat \& Büchel, 2015). Accordingly, an animal should extract the features from a known threat that most reliably predict danger, so as to respond appropriately to related stimuli that share those features. Research on stimulus generalization shows that learned behavior transfers to stimuli along dimensions of perceptual similarity (e.g. shapes and colors) and physical intensity (e.g. brightness or volume) (Dymond, Dunsmoor, Vervliet, Roche, \& Hermans, 2015). Generalization based on perceptual similarity is oftentimes strongest to stimuli that most closely resemble the trained conditioned stimulus (CS), and diminishes as similarity decreases (Guttman \& Kalish, 1956; Vervliet, Kindt, Vansteenwegen, \& Hermans, 2010). In contrast, behavioral generalization based on physical intensity is biased toward unreinforced stimuli that are more intense, and is typically accompanied by an asymmetrical shift in maximal responses from the CS to stimuli that possess an even more intense feature (Ghirlanda \& Enquist, 2003). Importantly, real-world stimuli are complex and contain a multitude of features that can be represented along dimensions of similarity or intensity. Moreover, the degree to which a potential threat is regarded as dangerous is likely based on the shared feature that best represents the learned threat stimulus along these dimensions. If so, this leads to a counterintuitive proposal that fear acquired to a positive emotional stimulus would lead to an asymmetrical fear to stimuli that are even more positive. Here, we tested whether fear conditioning to a face with a slight smile produces a counterintuitive shift in maximal fear responses to faces expressing even more happiness. 
Consider an example in which an individual has a terrifying encounter with a dog and then develops a widespread fear of dogs. Despite a general fear of dogs, the degree of fear experienced during a subsequent encounter with a large German Shepard would likely be greater than the fear experienced during an encounter with a little Pug (Dymond et al., 2015). In this scenario, the original threatening dog contained some features considered phylogenetically threatening (e.g. sharp teeth, darting movements, aggressive vocalizations) as well as typical dog-like features (e.g. floppy ears). When assessing the threat value of other dogs, the features most relevant to danger (e.g., size of its teeth) are likely prioritized over those less threat-relevant features (e.g., floppiness of its ears). According to fear preparedness theory (Ohman \& Mineka, 2001), the neural circuitry responsible for defensive behavior and fear phenomenology evolved to be selective toward these types of ecologically threatening features. Indeed, several studies have demonstrated that evolutionarily threat-relevant stimuli (e.g., angry faces, spiders) are more readily associated with an aversive outcome and take longer to extinguish than threat-irrelevant stimuli (Dunsmoor, Ahs, Zielinski, \& LaBar, 2014; Ohman \& Mineka, 2001; Seligman, 1970; but see Åhs et al., 2018 for a systematic review critiquing preparedness theory). Dunsmoor et al. $(2009 ; 2011)$ showed that fear generalization is likewise skewed toward modified face stimuli expressing even more fear than the original threat, in line with an intensity-bias toward more negative-relevant emotional stimuli.

Contrariwise, recent work suggests fear learning is not selectively enhanced to threatrelevant stimuli, per se, but extends to stimuli that are simply relevant to an animals' concerns. This can include goal-relevant (Stussi, Ferrero, Pourtois, \& Sander, 2019) and even positiverelevant stimuli (images of infants and erotica) (Stussi, Pourtois, \& Sander, 2018). These two prevailing accounts of how animals extract either threat-relevant or merely relevant features 
pertinent to detecting future threat make differentiable predictions as to which features of a stimulus an individual may generalize to: threat-relevant features (preparedness theory), or any salient features, regardless of valence (relevance detection hypothesis ; Frijda, 1988), including positive-relevant features.

As a strong test of the accounts described above, we compared response gradients to stimuli bearing features with ecologically divergent valence along dimensions of increasing intensity. Given the importance of avoiding inherently threatening stimuli in the natural world, it would perhaps be counterintuitive to expect fear to generalize as a function of the positivity of an emotional stimulus. Yet, if fear conditioning is selectively enhanced for both negative (Ohman \& Mineka, 2001) and positive CSs (Stussi et al., 2018) (as compared to affectively neutral CSs), then the same general mechanism might support fear generalization to unreinforced stimuli that share either positive or negative features with the CS. Participants in two experiments were fear conditioned to a face morphed along a dimension of either happiness (Experiment 1) or fear expression (Experiment 2). Fear conditioning was then followed by a fear generalization test to a gradient of faces morphed along the respective facial expression containing more or less emotional expression intensity than the original threat CS.

Given evidence supporting the capacity for enhanced fear learning to positive emotional stimuli (Stussi et al., 2018), as well as intensity-based accounts of stimulus generalization (Ghirlanda \& Enquist, 2003), we predicted fear responses would generalize as a function of emotional intensity, regardless of valence. Secondarily, we predicted that fear generalization along a dimension of positive emotional expression would diminish over the course of testing with unreinforced stimuli, whereas fear generalization along a dimension of threat-relevant emotional expressions would be maintained for a relatively longer duration over the course of 
testing. Finally, we predicted that participants who were fear conditioned to a slightly fearful face would retrospectively misremember the specific threat stimulus as exhibiting a more intense expression than it actually was (Experiment 2-in line with prior reports from Dunsmoor et al., 2009,2011 ), but that a memory bias would be less pronounced when the original threat was a positive emotional face (Experiment 1).

\section{METHOD}

Participants. Forty healthy adult participants (29 female: $M=25.30$ years, $S D=3.23$ ) participated in one of the two experiments described below. One participant failed to finish Experiment $1(n=19)$ and equipment failure occurred while running one participant in Experiment $2(\mathrm{n}=19)$. The target sample size was determined by a power analysis in G*Power 3 (Faul, Erdfelder, Lang, \& Buchner, 2007), using the effect size from prior research (Dunsmoor et al., 2009), and in accordance with field-standard number of participants for fear conditioning and generalization research (Dunsmoor et al., 2014; Stussi et al., 2018). Recruitment exclusionary criteria included a history of psychiatric, neurological, or major medical illnesses or current use of psychoactive medication. The study was approved by the Institutional Review Board at the University of Texas at Austin (IRB \#2017-02-0094).

Stimulus Set. A male face morphed along a gradient from neutral-to-happy was used as the stimulus set for Experiment 1 and the same male face morphed along a gradient from neutralto-fearful was used for Experiment 2. This identity was taken from the Ekman pictures of facial affect (Ekman \& Friesen, 1976) and was morphed along a continuum from neutral-to-happy and neutral-to-fearful (Kevin S. LaBar, Crupain, Voyvodic, \& McCarthy, 2003). Face morphs were positioned in a full-frontal orientation and cropped to include only the face, without hair, ears, or neckline. Images were normalized for contrast and luminance and appeared on a gray rectangular 
background. Participants were exposed to the same face identity during all experimental phases to ensure that only happy and fear expression and no other features related to actor identity (i.e., markings specific to an individual face such as moles or facial hair), were manipulated. Six faces along the happy continuum were used for Experiment 1: 100\% neutral, 11\% fear/88\% neutral, $33 \%$ fear $/ 66 \%$ neutral, $55 \%$ fear/44\% neutral, $77 \%$ fear $/ 2 \%$ neutral, and $100 \%$ fear; and six faces along the fearful continuum were used for Experiment 2: 100\% neutral, $11 \%$ happy/88\% neutral, $33 \%$ happy $/ 66 \%$ neutral, 55\% happy/44\% neutral, 77\% happy/2\% neutral, and 100\% happy. For Experiment 1, these stimuli are also labeled as SN, SH1, SH2, SH3, SH4, and SH5, respectively, where SN refers to 'Stimulus Neutral' and SH refers to 'Stimulus Happy.' Likewise, for Experiment 2, these stimuli were labelled as SN, SF1, SF2, SF3, SF4, and SF5, where SF refers to 'Stimulus Fear.' Hence, either the SH3 (Experiment 1) or SF3 (Experiment 2) served as the conditioned stimulus (CS+), and SN served as the unpaired CS (CS-) during fear conditioning. Importantly, both experiments used the same neutral face (SN) as the unpaired CS- during fear conditioning.

Skin conductance. Skin conductance was recorded using a BIOPAC MP160 system (BIOPAC Systems Inc.; Santa Barbara, CA) with pre-gelled electrodes attached to the hypothenar eminence of the palmar surface of the left hand. Skin conductance responses (SCR) for each phase of the study were calculated using Autonomate and hand-scored by the second author for validity and to check for potential artefacts (Green, Kragel, Fecteau, \& LaBar, 2014). Electrical Shock Procedures. An electrical shock (50 milliseconds) delivered to the right wrist served as the unconditioned stimulus for both experiments. Electrical shocks were delivered via electrodes connected to a stimulator (BIOPAC Systems Inc.; Santa Barbara, CA). The intensity (i.e. voltage) of the shock was calibrated separately for each participant in a 
stepwise manner, starting from a low barely perceptible setting and ending at a level that the participant rated as being "highly annoying and unpleasant, but not painful," based on the protocols of prior threat conditioning studies (Dunsmoor et al., 2014; K S LaBar, Gatenby, Gore, LeDoux, \& Phelps, 1998).

Task Design and Procedure. After obtaining informed consent and attachment of the electrodes, participants viewed a 5-minute video that served as a baseline washout prior to the start of the conditioning experiment. Videos were obtained from an episode of Norsk Slow TV (Niederer, 2017). Participants were told that the video was unrelated to the experiment and to simply pay attention for five minutes. Participants were told to turn off their phones.

During the fear-conditioning (FC) phase, participants were presented with both the CS+ (SH3; 55\% happy in Experiment 1, SF3; 55\% fearful in Experiment 2) and the CS- (SN; 100\% neutral in both experiments) 15 times each. Each face was presented for 4.5 seconds and was separated by an ITI that lasted 6 seconds. Faces were presented in a predetermined pseudorandom order and co-terminated with the US in 9 of the $15 \mathrm{CS}+$ presentations $(60 \% \mathrm{CS}+\mathrm{US}$ reinforcement rate). On each trial participants were instructed to indicate if the face was displaying either happiness (Experiment 1) or fear (Experiment 2) or not by pressing 1 (no) or 2 (yes) on the keyboard.

Following fear conditioning, participants had a five minute break during which another neutral video was shown. Participants then underwent the fear generalization test phase, during which they were presented with each of the 5 faces nine times (45 trials total). Each trial was separated by a 6 second ITI (Figure 3). The CS+ (SH3 or SF3) was intermittently paired with the US on $33 \%$ of generalization test trials ("steady-state" generalization test; Honig and Urcuioli 1981). These steady-state ("booster”' trials) reinforcement procedures are intended to 
extend the length of time over which responding can be measured and offset the effects of extinction and habituation (Honig \& Urcuioli, 1981; Lim \& Pessoa, 2008; Mednick \& Freedman, 1960). Generalization was tested either to faces along a continuum of happiness (Experiment 1) or fear (Experiment 2).

Statistical analysis. Statistical analyses were performed in python utilizing the pingoiun package (Vallat, 2018), the SciPy package (Jones, Oliphant, Peterson, et al., 2001), as well as R for mixed-effects regression modelling (lmer function in the lme4 library; Bates, Mächler, Bolker, \& Walker, 2015). Within each experiment, bootstrapped confidence intervals (CI; n boot $=5000)$ were computed to determine differences of SCR and reaction times between CS- and $\mathrm{CS}+$ during fear conditioning. Fear generalization SCRs and reaction times were analyzed using a 1x5 (gradient of face) repeated-measures analysis of variance (ANOVA). Mixed linear and polynomial regression models were performed on generalization SCRs within each experiment and tested for best fit using likelihood ratio tests. Across experiments, a Kruskal-Wallis test was used to investigate differences in retrospective CS+ identification. For temporal analysis of the generalization test, each participant's data was grouped into tertiles of 15 trials. We then

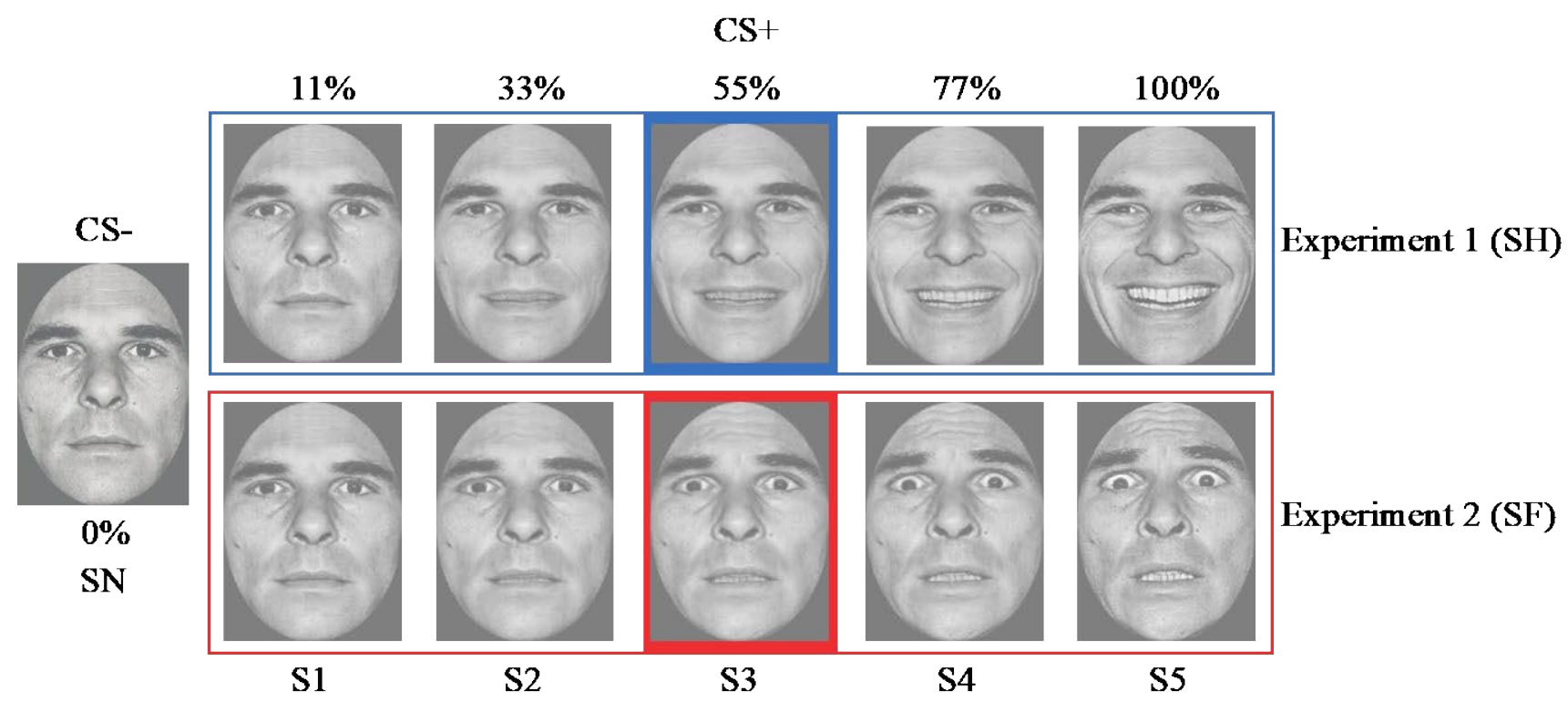


performed a binomial curve-fitting procedure using least-squares in SciPy to model SCRs across the stimulus-gradient for each participant in each tertile. Within each tertile, bootstrapped CIs (n boot $=5000)$ of correlations were computed to investigate the similarity of generalization gradients between experiments over time.

Figure 1. Stimulus dimensions. All participants in both experiments underwent initial fear conditioning to an unpaired CS- (a 100\% neutral face) and a CS+ (either 55\% happy face [Experiment 1] or 55\% fear face [Experiment 2]) paired with shock. Fear conditioning was followed by a generalization test, during which participants viewed the CS+ and unreinforced faces along a continuum morphed between a neutral face and the target emotion. Two morphed stimuli were expressed less emotion than the $\mathrm{CS}+$ and two faces expressed more emotion than the $\mathrm{CS}+$. $\mathrm{CS}=$ conditioned stimulus; $\mathrm{SH}=$ Stimulus Happy; $\mathrm{SF}=$ Stimulus Fear.

\section{RESULTS}

\section{Experiment 1: Fear generalization to positive emotional stimuli}

Conditioning. Success of conditioning was verified by heightened mean SCRs to the CS+ (55\% happy) versus CS- (100\% neutral) faces, 95\% CI $=[.074, .245]$ (Figure 2a). Moreover, emotion identification RTs were faster to the CS+ relative to the CS-95\% CI $=[-27.97,-169.68]$ (Figure 2b). As expected, participants rated the CS+ face as expressing happiness on $98 \%$ of trials, whereas the CS- face was rated as expressing happiness on just $1 \%$ of trials (Figure $2 \mathrm{c}$ ).

A.

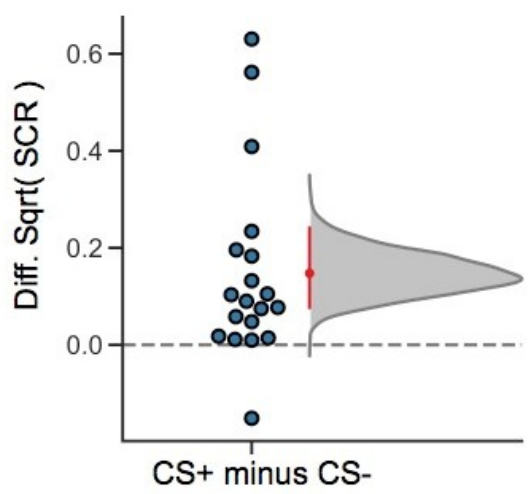

B.

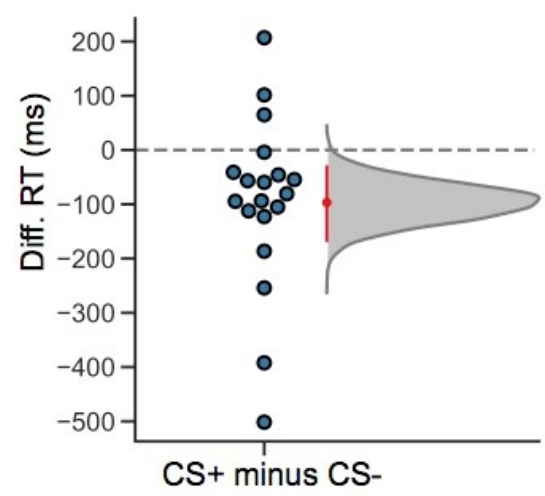

C.

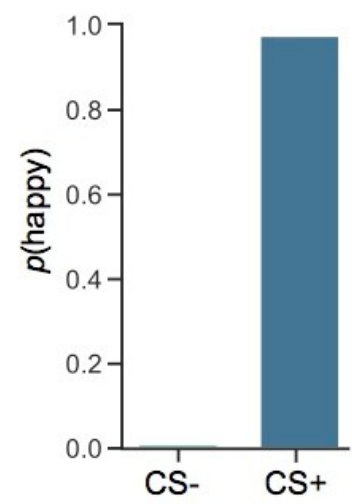


Figure 2: Fear conditioning results for Experiment 1. (a) Mean difference between CS+ and CSskin conductance responses (SCR) in microsiemens (uS). (b) Mean difference between CS+ and CS- reaction times (RTs). (c) Subjective face ratings (happy or not) between the CS- and CS+. Effect sizes are represented by bootstrap $95 \% \mathrm{CI}$.

Generalization test. A mixed-effects model with a quadratic (second-order) term was a better fit than one without, $\chi 2(1)=27.105, \mathrm{p}<0.0001$, suggesting that the SCR generalization curve was characterized by a peak between the $\mathrm{CS}+(\mathrm{SH} 3)$ and the highest intensity face (SH5). When the generalization task was segmented into tertiles, a mixed-effects model with a quadratic (second-order) term was a better fit than one without, $\chi 2(1)=0, p<0.9972$, a linear mixed-effects model best fit the second tertile, $\chi 2(1)=20.6, p<0.0001$, and a linear mixed-effects model best fit the third tertile, $\chi 2(1)=20.805, p<0.0001$ (see Supplementary tables for full model statistics). This demonstrates that intensity-based generalization toward the happier faces was most pronounced during early testing, but that asymmetric generalization to the happier faces diminished over the course of testing. Repeated-measures ANOVA confirmed a significant main effect for stimulus intensity value on SCR magnitude, $\mathrm{F}(4,72)=7.383, p<.001, \eta_{\mathrm{p}}^{2}=.291$. Post-hoc Bonferroni-corrected t-tests were performed and revealed significant differences between SH1 and SH4 $(\mathrm{P}=.003$, hedges $=.463)$, between $\mathrm{SH} 1$ and SH5 $(\mathrm{P}=.012$, hedges $=$ $.312)$, between SH2 and SH3 $(\mathrm{P}<.0001$, hedges $=.494)$, between $\mathrm{S} 2$ and $\mathrm{S} 4(\mathrm{P}=.005$, hedges $=$ $.361)$, between SH3 and SH5 $(\mathrm{P}=.013$, hedges $=.316)$ and between $\mathrm{S} 1$ and $\mathrm{S} 3(\mathrm{P}<.0001$, hedges $=.597)($ Figure 3a).

Reaction Times. A repeated-measures ANOVA revealed a main effect of stimulus type on reaction times, $\mathrm{F}(4,72)=14.041, \mathrm{P}<.0001, \eta_{\mathrm{p}}{ }^{2}=.438$, indicating that the fastest reaction times were elicited by the most intense happy face (SH5) and the slowest reaction times were observed for the more ambiguous stimuli (SH2 and SH3) (Figure 3b). 
Retrospective CS + identification. Following the session, participants were asked to identify which face stimulus had been paired with the US during the course of the experiment (Figure 3c). The majority of participants (12/19) correctly identified the SH3 (55\% happiness intensity) as the $\mathrm{CS}+$, whereas 1 participant misidentified the $\mathrm{SH} 2$ (33\% happiness intensity) and 6/19 participants misidentified the SH4 (77\% happiness intensity) as being the CS+.

Interestingly, these results map the curvilinear relationship across happiness intensity and SCRs during the generalization test.

Subjective face ratings. As expected, the $\mathrm{SH} 1$ and $\mathrm{SH} 2$ were rarely endorsed as expressing happiness, while $\mathrm{SH} 3, \mathrm{SH} 4$, and $\mathrm{SH} 5$ were consistently rated as expressing happiness (Figure 3d).

A.

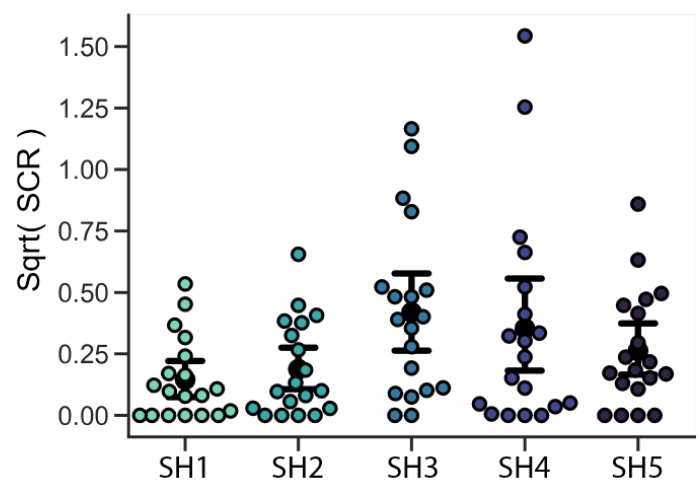

C.

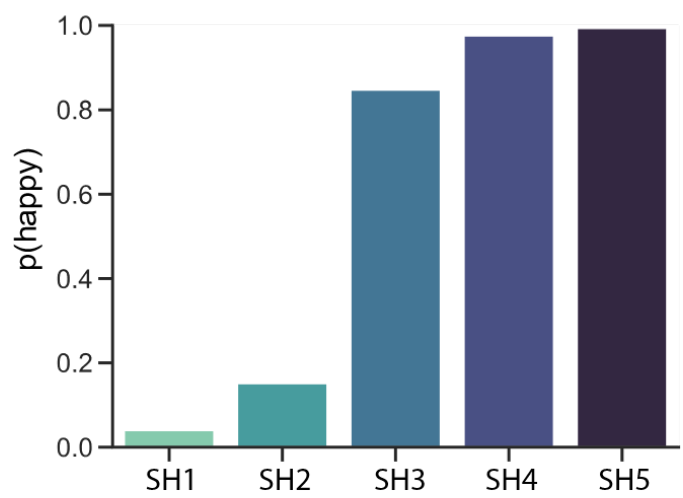

B.

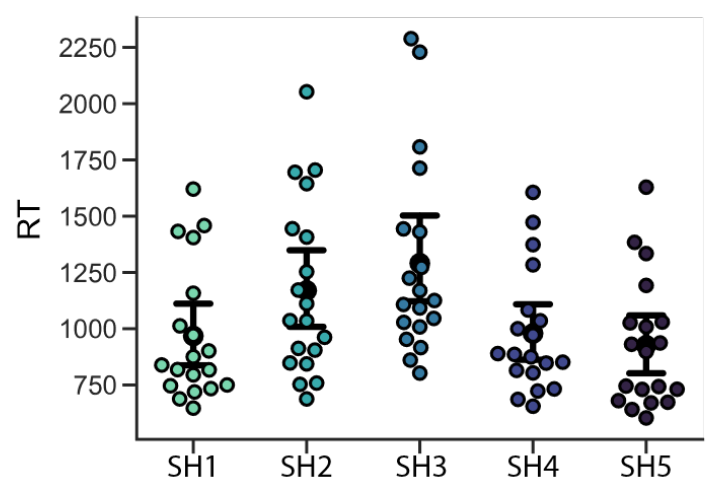

D.

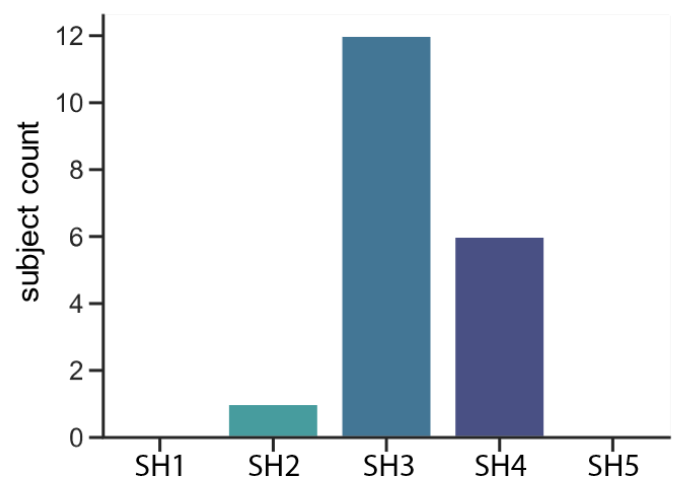


Figure 3: Fear generalization results for Experiment 1. (a) Mean skin conductance responses (SCR) in microsiemens (uS). (b) Mean reaction times (RTs). (c) Frequency of face selection for the retrospective CS+ identification task. (d) Subjective face ratings (happy or not) across generalization stimuli. Error bars reflect $95 \%$ bootstrap CI.

\section{Experiment 2: Fear generalization to negative emotional stimuli}

Conditioning. Conditioning was verified by heightened mean SCRs to the CS+ $(55 \%$ fear) versus CS- $(100 \%$ neutral) faces, $95 \% \mathrm{CI}=[0.36,0.53]$ (Figure 4a). Moreover, RTs were faster to the CS+ relative to the CS-, 95\% CI [-152.87, 0.29] (Figure 4b). As expected, participants rated the CS+ as expressing fear on $99 \%$ of trials, whereas the CS- was rated as expressing fear on less than $1 \%$ of trials (Figure $4 \mathrm{c}$ ).

A.

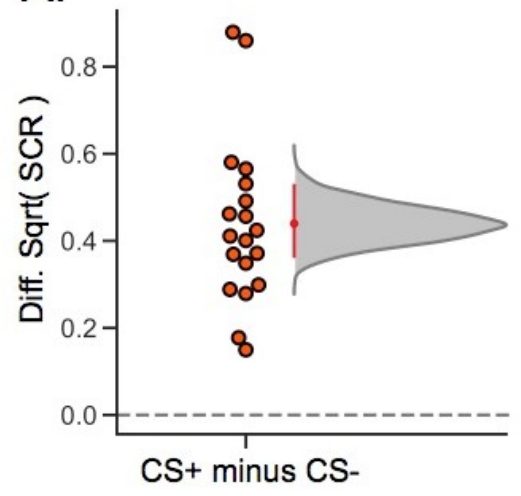

B.

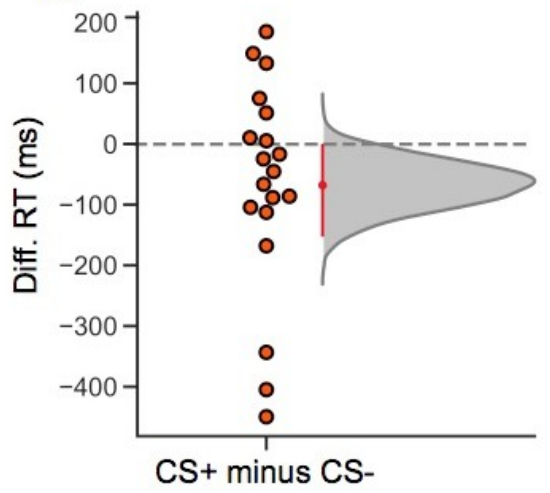

C.

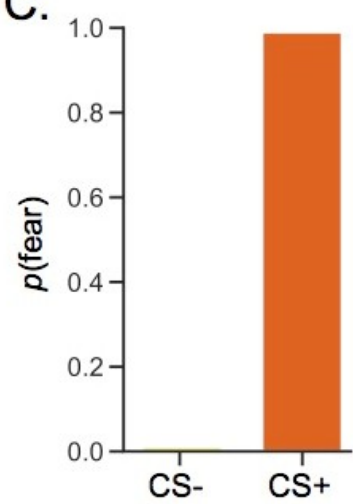

Figure 4: Fear conditioning results for Experiment 2. (a) Mean difference between CS+ and CSskin conductance responses (SCR) in microsiemens ( $\mathrm{uS}$ ). (b) Mean difference between CS+ and CS- reation times (RTs). (c) Subjective face ratings (fear or not) between the CS- and CS+. Effect sizes are represented by bootstrap $95 \% \mathrm{CI}$.

Generalization test. A linear mixed-effects model was a better fit than a quadratic (second-order) mixed-effects polynomial model, $\chi 2(1)=.6865, \mathrm{p}<0.40$, suggesting that the $\mathrm{SCR}$ generalization curve was characterized by a peak between the $\mathrm{CS}+(\mathrm{SH} 3)$ and the highest intensity face (SH5). When the generalization task was segmented into tertiles, a linear mixedeffects model best fit the first tertile, $\chi 2(1)=1.71, p<0.19$, a quadratic (second-order) mixed- 
effects polynomial model best fit the second tertile, $\chi 2(1)=0.36, p<0.55$, and a quadratic (secondorder) mixed-effects polynomial model best fit the third tertile, $\chi 2(1)=0.27, p<0.60$ (see Supplementary tables for full model statistics). Importantly, SCR peaked at the most intensely expressive fearful face morph within each phase, indicating that generalization persisted along a gradient of fear intensity despite repeated unreinforced exposure. Repeated-measures ANOVA confirmed a significant main effect for fear intensity value on SCR magnitude, $\mathrm{F}(4,72)=p<$ $.001, \eta_{\mathrm{p}}^{2}=.351$. Post-hoc Bonferroni-corrected t-tests were performed and revealed significant differences between SF1 and SF4 $(p=.003)$, SF2 and SF4 $(p=.031$, hedges $=.305)$, SF1 and SF5 $(p<.001$, hedges $=.492)$, SF2 and SF5 $(p<.001$, hedges $=.456)$, and SF3 and SF5 $(p<$ .001$, hedges $=.313)($ Figure 5a).

Reaction times. A repeated-measures ANOVA revealed a main effect of stimulus type on reaction time, $\mathrm{F}(4,72)=15.519, p<.0001, \eta_{\mathrm{p}}^{2}=.463$, indicating that the fastest reaction times were elicited by the most intense fear face (SF5) and the slowest reaction times were observed for the more ambiguous stimuli (SF1 and SF2) (Figure 5b).

Retrospective CS+ identification. Following the session, participants were asked to identify which face stimulus had been paired with the US during the course of the experiment (Figure 5c). Only 1 participant correctly identified the SF3 (55\% fear intensity) as the CS+, whereas 12/19 participants misidentified the SF4 (77\% fear intensity) and 6/19 misidentified the SF5 (100\% fear intensity) as being the CS+. Similarly to Experiment 1, these results map the relationship across fear intensity and SCRs during the generalization test. 
Subjective face ratings. As expected, SF1 was rarely endorsed as expressing fear, while SF2 was rated as expressing fear on $40 \%$ of the trials and SF3, SF4, and SF5 were rated as expressing fear on the majority of the trials (Figure $5 \mathrm{~d}$ ).

A.

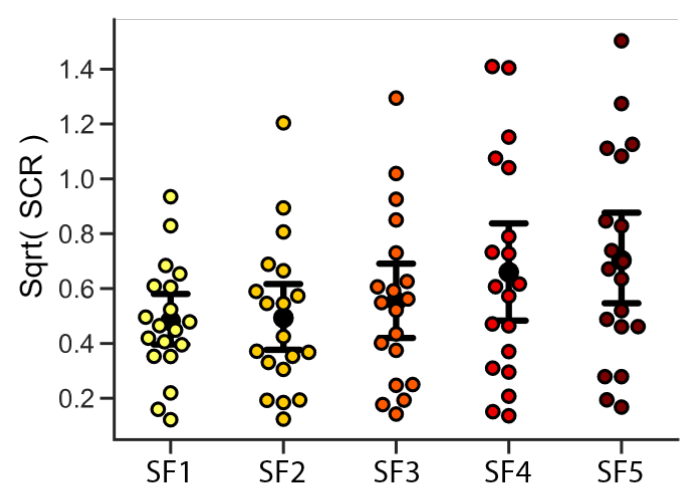

C.

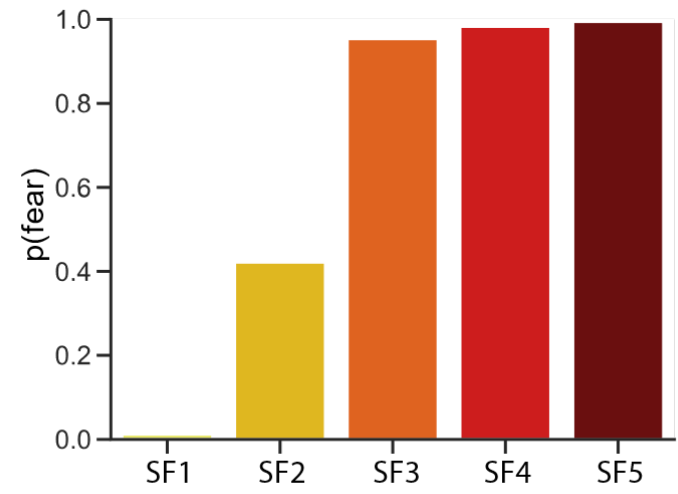

B.

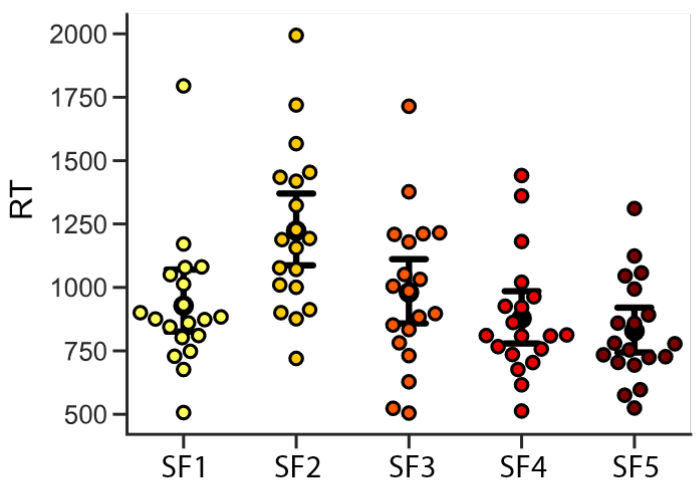

D.

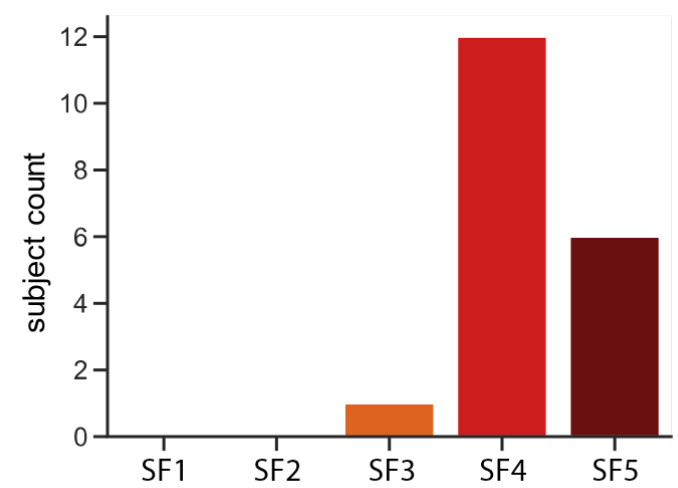

Figure 5: Fear generalization results for Experiment 2. (a) Mean skin conductance responses (SCR) in microsiemens (uS). (b) Mean reaction times (RTs). (c) Frequency of face selection for the retrospective CS+ identification task. (d) Subjective face ratings (fearful or not) across generalization stimuli. Error bars reflect $95 \%$ bootstrap CI.

\section{Comparisons across experiments}

Retrospective CS+ identification. In order to compare explicit generalization along a dimension of stimulus intensity across experiments, we performed a Kruskal-Wallis H-test on the retrospective CS+ identification scores. The Kruskal-Wallis H-test revealed a significant 
difference in CS+ identification between experiments, $\mathrm{H}(1)=15.520, p<.0001$, where participants were more likely to falsely identify a more intense stimulus as being the $\mathrm{CS}+$ in Experiment 2 (faces expressing fear) than in Experiment 1 (faces expressing happiness). This suggests that while some explicit generalization occurred in both experiments, this was enhanced in the experiment that implemented a gradient of stimuli along greater fear-relevance (Experiment 2).

Maintenance of fear generalization along a dimension increasing fear intensity. In order to test our hypothesis that fear generalization would be maintained for a longer duration in Experiment 2 (fearful faces) than Experiment 1 (happy faces), we parsed the generalization tests into tertiles for an Experiment-by-tertile analysis. We then performed a curve fitting procedure for each participant's SCRs across each tertile to acquire a best-fit second-degree polynomial model representing the curvature of SCR across the gradient of stimuli (S1 to S5) $\left(\mathrm{y}=a \mathrm{x}^{2}+b \mathrm{x}+\right.$ $c$; see Figure 6). In order to compare the obtained estimated curves between experiments across tertiles, we bootstrapped Pearson's correlations to estimate a 95\% CI of the relationship between experiments. Mean correlation for tertile 1 is $r=0.944,95 \% \mathrm{CI}=[0.34,0.99]$, for tertile 2 is $r=$ $0.29,95 \% \mathrm{CI}=[-0.80,0.84]$, for tertile 3 is $r=[0.18,0.97]$ (Figure 6$)$. The diminishment of the correlation between generalization curves over tertiles 2 and 3 provides evidence for divergence of generalization patterns over time between experiments. Specifically, while participants generalized during both experiments, generalization was greater and more persistent to fearful faces. 
Tertile 1
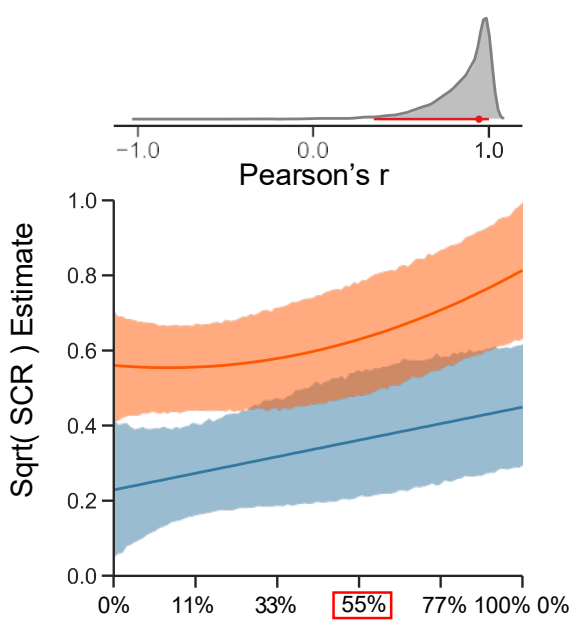

Tertile 2
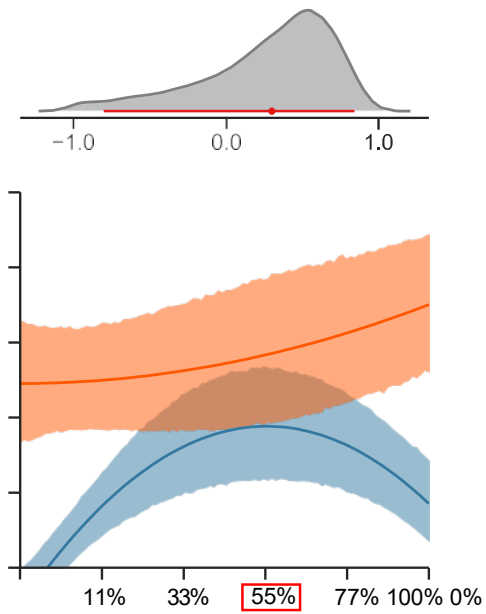

Tertile 3
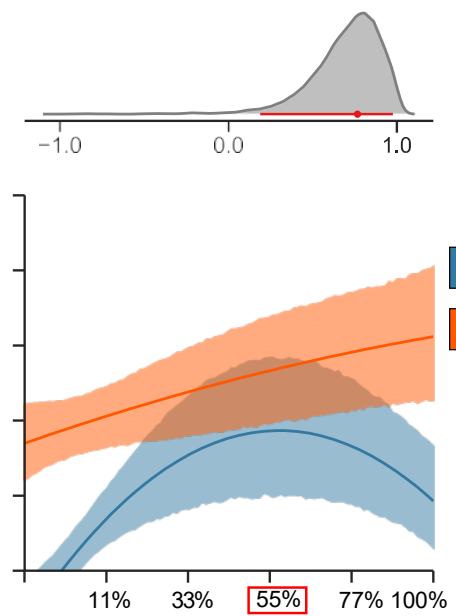

Exp. 1 (happy)

Exp. 2 (fearful)

Figure 6: Curve-fitting results for our experiment-by-tertile analysis of the generalization test. Estimated skin conductance responses (SCR) in microsiemens (uS) increases by a function of facial expression for both experiments in tertile 1 . The trend diverges for each experiment in tertiles 2 and 3, as reflected by lower correlations. Error bands reflect 95\% bootstrap CI.

\section{DISCUSSION}

The present study investigated whether fear conditioning to a stimulus containing a salient positive emotional feature would lead to a counterintuitive shift in fear generalization to a harmless but even more positive emotional stimulus. Specifically, we were interested in whether humans can base fear generalization on positive emotional features extracted from a learned threat, and how this is different than fear generalization based on an ecologically fear-relevant feature. We found biased fear generalization that transferred from a slightly expressive happy (Experiment 1) and fearful (Experiment 2) face to harmless face stimuli with an exaggerated emotional expression. These findings provide evidence that humans are capable of extracting positive emotional features of a learned threat to guide future fear responses to ambiguous threats. 
Asymmetric shifts in behavioral generalization toward stimuli that are more intense than the reinforced stimulus have been reported across species (Ghirlanda \& Enquist, 2003). These generalization biases are usually tested along perceptual dimensions of increasing volume, brightness, or size, but have also been reported along a negatively valenced dimension of fear expression in human fear conditioning (Dunsmoor et al., 2009, 2011). These strongly asymmetrical shifts tend to be robust when, during initial training, the CS + is intense and the CSis faint (reviewed in Ghirlanda \& Enquist, 2003), as this likely contributes to learning that 'intensity' is a key feature that discriminates reinforced from unreinforced stimuli.

Discrimination learning is likely a key factor in the present results as well, as subjects in both groups learned that emotional expression was the feature that discriminated the CS+ from the neutrally expressive CS-. Transposition to unreinforced stimuli that were even more unlike the CS- could explain the shift in maximal SCRs in both experiments. This would fit with elemental models of stimulus generalization that propose generalization is determined by the associative strength of those elements shared with the CS (McLaren \& Mackintosh, 2002). From this account, the valence of the features that discriminate threat from safety, whether positive or negative, is irrelevant.

Yet, there was an important difference in the maintenance of physiological arousal to the maximally fearful versus happy face across experiments that complicates a purely associative learning account. Whereas biased generalization to the happiest face diminished over the course of testing, generalization to the most fearful face persisted. A significant proportion of subjects also mistakenly identified the more intensely fearful face stimulus as the actual threat. This suggests that fear-relevance is an important factor in maintaining biased fear generalization, whereas experiences that disconfirm threat expectations to positive stimuli lead to a faster 
reduction in conditioned arousal and more accurate retrospective memory of threat. Interestingly, this distinction in fear generalization along positive and negative stimulus dimensions is not predicted from recent work showing selective fear conditioning and delayed extinction to positive emotional stimuli (Stussi et al., 2018).

These findings have some implications for understanding patterns of overgeneralization in psychiatric disorders. Laboratory research is beginning to reveal patterns of overgeneralization in anxiety and trauma-related disorders (Dunsmoor \& Paz, 2015). But it is important to consider that real-world stimuli associated with negative emotional experiences are complex and contain features that can be represented along multiple dimensions. As stimuli are rarely experienced in the exact same form, generalization requires transferring learning based on the quality and proportion of shared features across encounters with different stimuli. Thus, an adaptive learning system should extract those features that are most likely to portend danger in the future. Because not all negative experiences involve intrinsically dangerous stimuli (like snakes or spiders) or situations (like fear of heights), fear generalization in the real world may take seemingly unusual paths, leading to fear and avoidance of stimuli that are characteristically positive. For example, in social phobia, a fear of social situations, like a party, might scale with the size of the event. A fear of dogs might extend to even extremely cute and fluffy animals that share typically positive features (e.g., floppy ears, fur, wagging tail) with a dog that acted ferociously toward an individual in the past. In the context of the present experiment, a smile, typically used to build trust, might take on a negative association if someone's trust has been grossly violated in the past - thereby leading to a counterintuitive distrust of individuals with a great big smile. A better understanding of how behavior is influenced by the affective features extracted from a learned threat expands the explanatory power of fear conditioning models for affective disorders. 


\section{Author Contributions.}

MM and JED developed and designed the experiments. MM, AR, and AH conducted the data collection and analyzed the results. MM, AH, JED wrote the manuscript.

\section{Disclosure Statement.}

The authors declared that they had no conflicts of interest with respect to their authorship or the publication of this article.

\section{Data Availability Statement.}

The datasets generated during and/or analysed during the current study are available from the corresponding author on reasonable request.

\section{Acknowledgements.}

We thank our colleague, Sam Cooper, for statistical assistance. This work was supported by National Institutes of Health Grant R00 MH106719 to J.E.D.

\section{References}

Bradley, B. P., Mogg, K., Millar, N., Bonham-Carter, C., Fergusson, E., Jenkins, J., \& Parr, M. (1997). Attentional Biases for Emotional Faces. Cognition and Emotion, 11(1), 2542. https://doi.org/10.1080/026999397380014

Dunsmoor, J. E., Prince, S. E., Murty, V. P., Kragel, P. A., \& LaBar, K. S. (2011). Neurobehavioral mechanisms of human fear generalization. NeuroImage. https://doi.org/10.1016/j.neuroimage.2011.01.041

Dunsmoor, J. E., \& Paz, R. (2015). Fear Generalization and Anxiety: Behavioral and Neural Mechanisms. Biological Psychiatry. https://doi.org/10.1016/j.biopsych.2015.04.010

Dunsmoor, J. E., \& Murphy, G. L. (2014). Stimulus Typicality Determines How Broadly Fear Is Generalized. Psychological Science. https://doi.org/10.1177/0956797614535401

Dunsmoor, J. E., Mitroff, S. R., \& LaBar, K. S. (2009). Generalization of conditioned fear along a dimension of increasing fear intensity. Learning and Memory. https://doi.org/10.1101/lm.1431609 
Dunsmoor, J. E., \& LaBar, K. S. (2013). Effects of discrimination training on fear generalization gradients and perceptual classification in humans. Behavioral Neuroscience. https://doi.org/10.1037/a0031933

Dunsmoor, J. E., Ahs, F., Zielinski, D. J., \& LaBar, K. S. (2014). Extinction in multiple virtual reality contexts diminishes fear reinstatement in humans. Neurobiology of Learning and Memory. https://doi.org/10.1016/j.nlm.2014.02.010

Dymond, S., Dunsmoor, J. E., Vervliet, B., Roche, B., \& Hermans, D. (2015). Fear Generalization in Humans: Systematic Review and Implications for Anxiety Disorder Research. Behavior Therapy. https://doi.org/10.1016/j.beth.2014.10.001

Faul, F., Erdfelder, E., Lang, A.-G., \& Buchner, A. (2007). G*Power 3: A flexible statistical power analysis program for the social, behavioral, and biomedical sciences. Behavior Research Methods, 39, 175-191.

Frijda, N. H. (1988). The Laws of Emotion.

Ghirlanda, S., \& Enquist, M. (2003). A century of generalization. Animal Behaviour. https://doi.org/10.1006/anbe.2003.2174

Green, S. R., Kragel, P. A., Fecteau, M. E., \& LaBar, K. S. (2014). Development and validation of an unsupervised scoring system (Autonomate) for skin conductance response analysis. International Journal of Psychophysiology. https://doi.org/10.1016/j.ijpsycho.2013.10.015

Jones E, Oliphant E, Peterson P, et al. SciPy: Open Source Scientific Tools for Python, 2001-, http://www.scipy.org/ [Online; accessed 2019-09-19]. 
LaBar, K. S., Gatenby, J. C., Gore, J. C., LeDoux, J. E., \& Phelps, E. A. (1998). Human amygdala activation during conditioned fear acquisition and extinction: A mixed-trial fMRI study. Neuron. https://doi.org/10.1016/S0896-6273(00)80475-4

Lim, S. L., \& Pessoa, L. (2008). Affective Learning Increases Sensitivity to Graded Emotional Faces. Emotion. https://doi.org/10.1037/1528-3542.8.1.96

Lissek, S., Powers, A. S., McClure, E. B., Phelps, E. A., Woldehawariat, G., Grillon, C., \& Pine, D. S. (2005). Classical fear conditioning in the anxiety disorders: A meta-analysis. Behaviour Research and Therapy. https://doi.org/10.1016/j.brat.2004.10.007

Lissek, S., Biggs, A. L., Rabin, S. J., Cornwell, B. R., Alvarez, R. P., Pine, D. S., \& Grillon, C. (2008). Generalization of conditioned fear-potentiated startle in humans: Experimental validation and clinical relevance. Behaviour Research and Therapy, 46(5), 678-687. https://doi.org/10.1016/j.brat.2008.02.005

Lissek, S. (2012). Toward an account of clinical anxiety predicated on basic, neutrally mapped mechanisms of Pavlovian fear-learning: the case for conditioned overgeneralization. Depress and Anxiety, 29, 257-263. https://doi.org/10.1002/da.21922

Lissek, S., Bradford, D. E., Alvarez, R. P., Burton, P., Espensen-Sturges, T., Reynolds, R. C., \& Grillon, C. (2014). Neural substrates of classically conditioned fear-generalization in humans: A parametric fMRI study. Social Cognitive and Affective Neuroscience. https://doi.org/10.1093/scan/nst096

McLaren, I. P. L., \& Mackintosh, N. J. (2002). Associative learning and elemental representation: II. Generalization and discrimination. Animal Learning and Behavior, 30(3), 177-200. https://doi.org/10.3758/BF03192828 
Mednick, S. A., \& Freedman, J. L. (1960). Stimulus generalization. Psychological Bulletin. https://doi.org/10.1037/h0041650

Ohman, A., \& Mineka, S. (2001). Fears, Phobias, and Preparedness: Toward an Evolved Module of Fear and Fear Learning. Psychological Review, 108(3), 483-522. https://doi.org/10.1037/0033-295X.108.3.483

Niederer, S. (2017, May 19). Norsk SlowTV - Hurtigruten - Minutt for Minutt - TV Show Part 05 of 30. Retrieved from https://www.youtube.com/watch?v=noOFJZh4KbU\&t=60s

Schneider, F., Grodd, W., Weiss, U., Klose, U., Mayer, K. R., Nägele, T., \& Gur, R. C. (1997). Functional MRI reveals left amygdala activation during emotion. Psychiatry Research: Neuroimaging, 76(2-3), 75-82. https://doi.org/10.1016/S0925-4927(97)00063-2

Stussi, Y., Pourtois, G., Sander, D. (2018). Enhanced Pavlovian aversive conditioning to positive emotional stimuli. Journal of Experimental Psychology: General, 147, no. 6, p. 905-923.

Stussi, Y., Ferrero, A., Pourtois, G., \& Sander, D. (2019). Achievement motivation modulates Pavlovian aversive conditioning to goal-relevant stimuli. Npj Science of Learning, 4(1), 4. https://doi.org/10.1038/s41539-019-0043-3

Vallat, R. Pingouin: statistics in Python. J. Open Source Softw. 3, 1026 (2018).

Zhu, X. ru, \& Luo, Y. jia. (2012). Fearful faces evoke a larger C1 than happy faces in executive attention task: An event-related potential study. Neuroscience Letters. https://doi.org/10.1016/j.neulet.2012.08.011 